\title{
RESPONSE OF DIFFERENT PROMISING RICE GENOTYPES TO VARIOUS NITROGEN LEVELS.
}

Metwally, T. F. ; E. E. Gewaily ; E. S. Naeem and M. M. El-Malky

Rice Res. and Training Center, Field Crops Res. Inst., Agric. Res. Center, Sakha 33717 - Kafr El-Sheikh, Egypt.

\begin{abstract}
Two field experiments were conducted at the Experimental Farm of Rice Research and Training Center, Sakha, Kafer El-Sheikh, Egypt during the rice growing seasons of 2009 and 2010 to evaluate the response of different rice genotypes namely, GZ8455, GZ8450, GZ7576, GZ8479, GZ8126, GZ7769, GZ7764 and GZ6522 (Sakha105) to various nitrogen levels (0, 40, 80, 120, 160, 200, 240, 280 and $320 \mathrm{Kg} \mathrm{N} \mathrm{ha}^{-1}$ ). Yield and yield attributes were determined at maturity. A wide variation in nitrogen response was observed in the eight rice genotypes. GZ6522 produced the highest grain yield followed by GZ8455 and GZ8126. Grain yield and most of yield attributes generally increased with increasing nitrogen levels up to 160 $\mathrm{kg} \mathrm{N} \mathrm{ha}^{-1}$.

Keywords: Rice Genotypes Nitrogen Levels
\end{abstract}

\section{INTRODUCTION}

Rice (Oryza sativa L.) is one of the most important cereal crops of the world, grown in wide range of climatic zones, to nourish the mankind. It is the staple food for more than three billion people that is over half of the world's total population (FAO, 2004).

Nitrogen is clearly an extremely important and essential element, and continuing research has constantly revealed more and more facets apart from its well known roles in plant metabolism and growth. Given the importance of nitrogen fertilization on the grain yield of rice crop, it is necessary to know what the best dose needed for each variety as well as its influence on components of yield. In many parts of the world, $\mathrm{N}$ fertilizer recommendations continue to follow a prescriptive approach using generic models of economic response, often without regard to site-specific variations in crop $\mathrm{N}$ requirement (Meisinger et al., 2008). The efficiency of nitrogen used by rice plant is central to its overall yield potential, and efforts to improve its potential must be guided by a thorough understanding of the processes that govern $\mathrm{N}$ use efficiency. Improvements in efficiency of nitrogen are also needed to reduce $\mathrm{N}$-fertilizer requirements.

Shaiful Islam et al. (2009) reported that by applying proper dose of nitrogen lead to save money and can also keep our environment sound. Moreover, the heavy use of fertilizer affects the soil and also the environment through the residual effect of fertilizer. Selection of the most appropriate level of nitrogen fertilization is a major concern of economic viability of crop production and the impact of agriculture. Metwally et al. (2010) studied the physio-morphological behavior of twenty one Egyptian rice genotypes under low and high nitrogen application. He found that There was a wide variation in Agronomic Nitrogen Use Efficiency (ANUE) among genotypes under low and high nitrogen levels. 
Metwally, T. F. et al.

This current research aims to study the response of several promising rice genotypes to different nitrogen levels to identify the actual requirement for each rice entry under study.

\section{MATERIALS AND METHODS}

Two field experiments were conducted at the Experimental Farm of Rice Research and Training Center, Sakha, Kafer El-Sheikh, Egypt during the rice growing seasons of 2009 and 2010. A total of eight rice entries were used to evaluate the suitable requirement of each entries for nitrogen. The origin and parentage of tested genotypes (GZ8455, GZ8450, GZ7576, GZ8479, GZ8126, GZ7769, GZ7764 and GZ6522) were presented in Table 1. Soil was clay and some basic properties of the soil at the experimental sit are shown in Table 2.

Treatments were laid out in a split plot design which replicated four times. Main plots treatments received rice entries, while nitrogen levels were located in sub plots. Fertilizer-N level treatments $0,40,80,120,160,200$, 240,280 and $320 \mathrm{~kg} \mathrm{~N}^{-1}$ were applied as form urea $(46.5 \% \mathrm{~N})$ in two equal split applications, the first dose as basal while the second dose after 30 days from transplanting.

The seed rate $96 \mathrm{~kg}$ seed ha-1 was used. Clean seeds of each genotype with at $90 \%$ germination were soaked in water for 24 hours and incubated for 48 hours. Pre-germinated seeds of rice genotypes were sown in May $10^{\text {th }}$ in both seasons. Seedling at 30 days old (3-4 seedling hill $\left.{ }^{-1}\right)$ were transplanted at $20 \times 20 \mathrm{~cm}$ distance between hills and rows. The plot size was kept $4 \times 3 \mathrm{~m}$. Phosphorus at the rate of $36 \mathrm{~kg} \mathrm{P}_{2} \mathrm{O}_{5} \mathrm{ha}^{-1}$ was applied as basal application during soil preparation. $\mathrm{ZnSO}_{4}$ containing $22 \%$ zinc was applied at $24 \mathrm{~kg}$ per hectare just before transplanting to eliminate zinc deficiency. After transplanting, $5 \mathrm{~cm}$ water depth was maintained in the experimental plots. Weeds were chemically controlled as recommended. Insects and diseases intensively controlled through the rice season to avoid any yield loss. . Ten days before harvest, the plots were drained to facilitate harvesting.

At harvest, number of panicle hill-1, panicle length, panicle weight, number of grains panicle-1 ${ }^{-1}$ sterility percentage, 1000-grain weight, grain yield and straw yield were recorded. Harvest index and agronomic nitrogen use efficiency (ANUE) was calculated.

Agronomic nitrogen use efficiency (ANUE) was computed according to Saleque et al.(2004) as follows:

$$
\text { ANUE }=\frac{\text { (Grain yield in fertilized plot } \mathbf{~ k g} \text { ) }-(\text { Grain yield in unfertilized plot } \mathbf{~ k g} \text { ) }}{\text { (Quantity of nutrient applied) } \mathbf{k g}}
$$

Data were analyzed by analysis of variance (ANOVA) in a split plot according to the procedure outlined by Gomez and Gomez (1984). Significant difference means were separated at $\mathrm{P}<0.05$ by the least significant difference (LSD) test. Statistical analyses were made with commercial software. 
Table 1: Origin and parentage of tested genotypes.

\begin{tabular}{|l|l|c|c|}
\hline Entry & Parentage & Origin & Group \\
\hline GZ8455 & GZ5603-3-3-2-1/Kanto51 & Egypt & Japonica \\
GZ8450 & GZ5603-3-2-2-1/RYNG Song 14 & Egypt & Japonica \\
GZ7576 & GZ5418/Milyang 79 & Egypt & Japonica \\
GZ8479 & GZ6214-4-1-1-1-1/Empssic 104 & Egypt & Japonica \\
GZ8126 & GZ5830-63-1-2/GZ5963-1-2-1-1 & Egypt & Japonica \\
GZ7769 & GZ5385-29-3-2/Akiyutaka & Egypt & Japonica \\
GZ7764 & GZ5320-5-1-1/Norin22 & Egypt & Japonica \\
GZ6522 & GZ5581-46-3/GZ4316-7-1-1 & Egypt & Japonica \\
\hline
\end{tabular}

Table 2: Some physical and chemical properties of the soil at the experimental site during 2009 and 2010 season.

\begin{tabular}{|l|c|c|}
\hline Soil properties & $\mathbf{2 0 0 9}$ & $\mathbf{2 0 1 0}$ \\
\hline Mechanical analysis & 54.06 & 55.80 \\
Clay \% & 30.64 & 31.50 \\
Silt \% & 15.30 & 13.20 \\
Sand \% & Clay & Clay \\
Textere & & \\
Chemical analysis & 1.45 & 1.60 \\
Organic matter \% & 540.00 & 570.00 \\
Total nitrogen , mg kg ${ }^{-1}$ & 15.00 & 17.00 \\
Available P, mg kg & \\
pH (1:2.5 soil suspension) & 8.10 & 8.19 \\
$\mathrm{EC} \mathrm{dS}^{-1}$ (soil paste) & 2.95 & 2.87 \\
$\mathrm{Soluble}^{-1}$ cations, meq. $\mathrm{L}^{-1}:$ & & \\
$\mathrm{Ca}^{++}$ & 9.50 & 10.00 \\
$\mathrm{Mg}^{++}$ & 3.94 & 3.98 \\
$\mathrm{~K}^{+}$ & 1.76 & 1.80 \\
$\mathrm{Na}^{+}$ & 14.80 & 13.20 \\
$\mathrm{Solube}^{+}$anions, meq. $\mathrm{L}^{-1}$ & & \\
$\mathrm{CO}_{3}{ }^{-1}$ & 0.00 & 0.00 \\
$\mathrm{HCO}_{3}^{-}$ & 6.00 & 6.75 \\
$\mathrm{Cl}^{-}$ & 8.30 & 8.44 \\
$\mathrm{SO}_{4}=$ & 15.7 & 13.79 \\
$\mathrm{Available} \mathrm{micronutrients} \mathrm{ppm}_{\mathrm{Fe}^{++}}$ & & \\
$\mathrm{Zn}^{++}$ & 6.10 & 5.80 \\
$\mathrm{Mn}^{++}$ & 1.10 & 1.05 \\
\hline
\end{tabular}

\section{RESULTS AND DISCUSSION}

Number of panicles hill-1

Number of panicles hill ${ }^{-1}$ was markedly influenced by different genotypes and various nitrogen levels as well as their interaction Table (3). Among the genotypes, GZ6522 significantly produced higher panicles number hill-1 than the other ones. GZ7764 recorded the lowest number of panicles hill-1. In the same Table, increasing nitrogen levels from 0 up to 160 $\mathrm{kg} \mathrm{N} \mathrm{ha}^{-1}$ significantly increased number of panicles hill-1. Moreover, there was a gradual reduction in number of panicles due to increasing nitrogen levels from 160 up to $320 \mathrm{~kg} \mathrm{~N} \mathrm{ha}^{-1}$. These findings are hold fairly true in the 
two seasons under study. The increases in number of panicles under $160 \mathrm{~kg}$ $\mathrm{N}$ ha-1 might be due to more availability of nitrogen that played a vital role during initiation of panicles while adding $\mathrm{N}$ more than $160 \mathrm{~kg} \mathrm{~N} \mathrm{ha}^{-1}$ increase the unproductive tillers due to increase vegetative growth period consequently decrease the productive tillers. These results are in accordance to the findings of Chaturvedi (2005) and Metwally et al. (2010).

Data in Table 4 revealed that the highest number of panicles ha-1 was obtained when GZ 6522 was fertilized by $160 \mathrm{~kg} \mathrm{~N}^{-1}$ in 2009 and 2010 seasons followed by the application of $200 \mathrm{~kg} \mathrm{~N} \mathrm{ha}^{-1}$ to the same entry in 2010 season. On the other hand the lowest number of panicles hill ${ }^{-1}$ was produced from GZ 7764 under unfertilized plots in the two seasons.

Panicle length (cm):

Data in Table 3 indicated that there were significant differences among both tested entries and $\mathrm{N}$ levels in panicle length. The tallest panicle was produced by GZ8455 followed by GZ6522, while GZ7764 produced the shortest panicle in both seasons. This variation in panicle length could be due to genetic background. The raising level of $\mathrm{N}$ up to $320 \mathrm{~kg} \mathrm{~N} \mathrm{ha}^{-1}$ significantly increased panicle length.

Table 3; Number of panicles hill- $^{-1}$, panicle length, panicle weight, number of grains panicle ${ }^{-1}$ and sterility \% of rice entries as affected by nitrogen levels in 2009 and 2010 seasons.

\begin{tabular}{|c|c|c|c|c|c|c|c|c|c|c|}
\hline \multirow[t]{2}{*}{ Treatment } & \multicolumn{2}{|c|}{$\begin{array}{c}\text { No. of } \\
\text { panicles hill }\end{array}$} & \multicolumn{2}{|c|}{$\begin{array}{c}\text { Panicle length } \\
\text { cm }\end{array}$} & \multicolumn{2}{|c|}{$\begin{array}{l}\text { Panicle } \\
\text { weight g }\end{array}$} & \multicolumn{2}{|c|}{$\begin{array}{l}\text { No. of grains } \\
\text { panicle }^{-1}\end{array}$} & \multicolumn{2}{|c|}{ Sterility \% } \\
\hline & 2009 & 2010 & 2009 & 2010 & 2009 & 2010 & 2009 & 2010 & 2009 & 2010 \\
\hline Entries: & & & & & & & & & & \\
\hline & 20.92 & 20.10 & 22.53 & 22.65 & 2.86 & 3.51 & 142.85 & 153.19 & 6.78 & 6.06 \\
\hline GZ84 & 20.03 & 21.22 & 20.82 & 21.58 & 2.49 & 3.43 & 124.41 & 145.12 & 5.40 & 5.32 \\
\hline & 3.93 & 19.49 & 20.71 & 21.21 & 2.43 & 3.39 & 108.16 & 124.10 & & 4.89 \\
\hline GZ8479 & 20.84 & 20.15 & 20.60 & 19.77 & 2.97 & 3.28 & 119.89 & 121.03 & 6.88 & 8.18 \\
\hline GZ8126 & 20.39 & 20.61 & 20.34 & 20.72 & 2.57 & 3.41 & 140.73 & 143.66 & 3.75 & 2.93 \\
\hline GZ7769 & 19.62 & 20.24 & 19.65 & 20.59 & 2.79 & 3.40 & & & 3.77 & 1.98 \\
\hline GZ7764 & 17.52 & 18.33 & 19.58 & 19.55 & 2.52 & 3.40 & 114.96 & 125. & & .55 \\
\hline GZ6522 & 22.65 & 23.13 & 22.13 & 21.11 & 3.05 & 3.67 & 141.72 & 150. & .77 & .14 \\
\hline LSD at 0.05 & 0.90 & 0.88 & 0.390 & 0.30 & 0.06 & 0.08 & 2.18 & & & 0.21 \\
\hline N kg ha-1 : & & & & & & & & & & \\
\hline 0 & 14.731 & 16.00 & 18.79 & 18.68 & 2.38 & 2.96 & 94.48 & & 3.00 & 2.98 \\
\hline 40 & 7.43 & 18.03 & 19.36 & 19.46 & 2.50 & 3.11 & 112.44 & 12 & & 3.50 \\
\hline 80 & 20.05 & 20.34 & 19.63 & 20.05 & 2.61 & 3.44 & 121.77 & 134 & 3.74 & 3.65 \\
\hline 120 & 22.25 & 22.56 & 20.09 & 20.20 & 2.79 & 3.57 & 134.34 & 144.85 & 3.72 & 3.98 \\
\hline & 23.61 & 23.63 & 20.93 & 21.09 & 2.94 & 3.74 & 148.30 & 152.60 & 3.96 & 4.21 \\
\hline 200 & 22.32 & 22.10 & 21.59 & 21.65 & 3.02 & 3.69 & 146.72 & 154.07 & 4.37 & 4.97 \\
\hline 240 & 21.00 & 21.08 & 21.82 & 21.97 & 2.84 & 3.60 & 140.66 & 146.91 & 5.41 & 5.85 \\
\hline 280 & 20.12 & 20.09 & 22.24 & 22.20 & 2.72 & 3.47 & 133.37 & 137.97 & 6.96 & 7.08 \\
\hline 320 & 19.52 & 19.85 & 22.74 & 22.79 & 2.59 & 3.36 & 128.70 & 134.62 & 8.88 & 8.83 \\
\hline LSD at 0.05 & 0.69 & 0.77 & 0.25 & 0.39 & 0.02 & 0.11 & 1.50 & 3.77 & 0.155 & 0.27 \\
\hline Interaction & $\star *$ & $*$ & $*$ & $\star \star$ & $\star * *$ & $\star *$ & * & * & ** & ** \\
\hline
\end{tabular}

The effect of the interaction between rice genotypes and nitrogen levels on panicle length was significant in both seasons (Table 5). The tallest panicle was found when GZ6522 fertilized with $320 \mathrm{~kg} \mathrm{~N} \mathrm{ha}^{-1}$, on the other hand the shortest one produced by GZ7576 when nitrogen was not applied. 
Table 4: Number of panicles hill-1 as affected by the interaction between rice entries and nitrogen levels in 2009 and 2010 seasons.

\begin{tabular}{|c|c|c|c|c|c|c|c|c|c|c|}
\hline \multirow{2}{*}{ Season } & \multirow{2}{*}{ Entry } & \multicolumn{9}{|c|}{ N kg ha-1 } \\
\hline & & 0 & 40 & 80 & 120 & 160 & 200 & 240 & 280 & 320 \\
\hline \multirow{9}{*}{2009} & GZ8455 & 15.00 & 18.00 & 23.00 & 24.67 & 25.33 & 22.33 & 21.00 & 19.67 & 19.33 \\
\hline & GZ8450 & 13.33 & 17.33 & 20.33 & 21.33 & 22.33 & 23.00 & 21.67 & 21.00 & 20.00 \\
\hline & GZ7576 & 13.00 & 15.33 & 18.67 & 23.33 & 22.00 & 20.67 & 19.67 & 19.10 & 18.67 \\
\hline & GZ8479 & 15.00 & 18.44 & 21.34 & 23.00 & 24.88 & 23.41 & 22.00 & 20.11 & 19.46 \\
\hline & GZ8126 & 17.00 & 18.67 & 19.33 & 20.33 & 24.33 & 24.10 & 20.67 & 19.87 & 19.27 \\
\hline & GZ7769 & 13.50 & 16.87 & 19.33 & 23.67 & 23.33 & 21.43 & 20.00 & 19.53 & 18.97 \\
\hline & GZ7764 & 12.33 & 14.67 & 17.00 & 18.67 & 20.34 & 19.00 & 18.83 & 18.53 & 18.33 \\
\hline & GZ6522 & 18.67 & 20.17 & 21.43 & 23.00 & 26.33 & 24.67 & 24.20 & 23.20 & 22.19 \\
\hline & LSD at 0.05 & \multicolumn{9}{|c|}{2.01} \\
\hline \multirow{9}{*}{2010} & GZ8455 & 14.67 & 16.50 & 21.83 & 23.00 & 24.33 & 21.72 & 20.11 & 20.00 & 18.78 \\
\hline & GZ8450 & 16.50 & 20.33 & 21.50 & 22.17 & 23.78 & 22.83 & 22.23 & 21.00 & 20.67 \\
\hline & GZ7576 & 15.83 & 16.56 & 20.00 & 22.50 & 23.17 & 21.12 & 20.33 & 16.17 & 19.78 \\
\hline & GZ8479 & 17.33 & 19.67 & 20.83 & 22.67 & 21.50 & 20.11 & 20.33 & 19.60 & 19.33 \\
\hline & GZ8126 & 16.17 & 18.11 & 19.78 & 23.67 & 24.39 & 23.28 & 20.66 & 20.17 & 19.33 \\
\hline & GZ7769 & 14.87 & 17.64 & 20.11 & 23.00 & 23.67 & 22.22 & 20.67 & 20.50 & 19.56 \\
\hline & GZ7764 & 13.67 & 15.33 & 18.00 & 19.44 & 21.33 & 19.87 & 19.53 & 19.33 & 18.52 \\
\hline & GZ6522 & 19.00 & 20.17 & 20.67 & 24.11 & 26.89 & 25.65 & 24.83 & 23.99 & 22.90 \\
\hline & LSD at 0.05 & \multicolumn{9}{|c|}{2.21} \\
\hline
\end{tabular}

Table 5: Panicle length as affected by the interaction between rice entries and nitrogen levels in 2009 and 2010 seasons.

\begin{tabular}{|c|c|c|c|c|c|c|c|c|c|c|}
\hline \multirow{2}{*}{ Season } & \multirow[t]{2}{*}{ Entry } & \multicolumn{9}{|c|}{ N kg ha-1 } \\
\hline & & 0 & 40 & 80 & 120 & 160 & 200 & 240 & 280 & 320 \\
\hline \multirow{9}{*}{2009} & GZ8455 & 20.57 & 22.49 & 22.13 & 22.13 & 22.53 & 22.97 & 23.21 & 23.53 & 23.27 \\
\hline & GZ8450 & 18.23 & 18.23 & 19.03 & 19.69 & 20.43 & 22.63 & 22.43 & 23.13 & 23.62 \\
\hline & GZ7576 & 17.13 & 17.77 & 18.37 & 19.91 & 23.24 & 22.25 & 22.21 & 22.53 & 23.00 \\
\hline & GZ8479 & 18.99 & 19.13 & 19.63 & 20.23 & 21.13 & 21.61 & 21.63 & 20.93 & 22.13 \\
\hline & GZ8126 & 19.03 & 19.23 & 19.23 & 19.33 & 19.63 & 20.63 & 21.77 & 21.78 & 22.47 \\
\hline & GZ7769 & 17.43 & 18.43 & 18.83 & 19.16 & 19.43 & 19.97 & 20.03 & 21.50 & 22.07 \\
\hline & GZ7764 & 18.47 & 18.93 & 19.03 & 19.23 & 19.43 & 20.03 & 20.23 & 20.33 & 20.53 \\
\hline & GZ6522 & 20.43 & 20.63 & 20.73 & 21.03 & 21.63 & 22.63 & 23.03 & 24.23 & 24.83 \\
\hline & LSD at 0.05 & \multicolumn{9}{|c|}{0.76} \\
\hline \multirow{9}{*}{2010} & GZ8455 & 20.55 & 21.35 & 22.60 & 22.15 & 22.80 & 23.21 & 23.36 & 23.85 & 23.98 \\
\hline & GZ8450 & 19.05 & 20.11 & 20.75 & 20.15 & 21.25 & 22.72 & 23.00 & 23.60 & 23.60 \\
\hline & GZ7576 & 19.40 & 20.05 & 20.00 & 19.55 & 22.20 & 22.05 & 22.25 & 21.90 & 23.50 \\
\hline & GZ8479 & 18.05 & 17.60 & 18.85 & 20.75 & 21.30 & 21.00 & 20.75 & 19.70 & 19.96 \\
\hline & GZ8126 & 18.60 & 19.05 & 19.95 & 20.53 & 19.65 & 21.10 & 21.95 & 22.40 & 23.30 \\
\hline & GZ7769 & 17.40 & 19.70 & 21.00 & 19.65 & 20.10 & 21.40 & 21.91 & 22.00 & 22.16 \\
\hline & GZ7764 & 17.70 & 17.55 & 18.05 & 19.10 & 20.04 & 20.15 & 20.50 & 21.30 & 21.60 \\
\hline & GZ6522 & 18.70 & 20.30 & 19.20 & 19.70 & 21.35 & 21.60 & 22.05 & 22.85 & 24.25 \\
\hline & LSD at 0.05 & \multicolumn{9}{|c|}{1.09} \\
\hline
\end{tabular}

\section{Panicle weight (g):}

Panicle weight of the eight tested entries was influenced by different nitrogen levels as well as the interaction (Table 3). Data showed that in both seasons the heaviest panicle was produced by GZ6522. The application of any of tested $\mathrm{N}$ levels caused an increase in panicle weight compared to control. Panicle weight was significantly increased by increasing nitrogen levels up to 200 and $160 \mathrm{~kg} \mathrm{~N} \mathrm{ha}^{-1}$ in the 2009 and 2010 seasons, respectively, but it was significantly decreased by increasing nitrogen levels 
from 200 up to $320 \mathrm{~kg} \mathrm{~N} \mathrm{ha}^{-1}$ in the first season and from 160 up to $320 \mathrm{~kg} \mathrm{~N}$ $\mathrm{ha}^{-1}$ in the second season.

Regarding to the interaction effect of genotypes and nitrogen levels, data presented in Table 6 indicated that GZ6522 produced the highest values of panicle weight when fertilized with $160 \mathrm{~kg} \mathrm{~N} \mathrm{ha}^{-1}$ in both seasons. While the lowest panicles weight was produced from GZ 8450 or GZ 8455 under control treatment in 2009 and 2010 seasons, respectively. Addition of suitable nitrogen fertilizer might improve the photosynthetic capacity during grain filling period and resulted in heaviest panicle and grains. These findings are in confirm with those obtained by Singh et al. (2004).

Table 6: Panicle weight as affected by the interaction between rice entries and nitrogen levels in 2009 and 2010 seasons.

\begin{tabular}{|c|c|c|c|c|c|c|c|c|c|c|}
\hline \multirow{2}{*}{ Season } & \multirow{2}{*}{ Entry } & \multicolumn{9}{|c|}{$\mathrm{N} \mathrm{kg} \mathrm{ha}^{-1}$} \\
\hline & & 0 & 40 & 80 & 120 & 160 & 200 & 240 & 280 & 320 \\
\hline \multirow{9}{*}{2009} & GZ8455 & 2.49 & 2.69 & 2.75 & 2.85 & 3.23 & 3.09 & 3.01 & 2.98 & 2.63 \\
\hline & GZ8450 & 1.88 & 2.13 & 2.43 & 2.59 & 2.80 & 2.96 & 2.74 & 2.56 & 2.28 \\
\hline & GZ7576 & 2.15 & 2.30 & 2.38 & 2.51 & 2.51 & 2.62 & 2.63 & 2.38 & 2.38 \\
\hline & GZ8479 & 2.64 & 2.83 & 2.87 & 3.31 & 3.37 & 3.10 & 2.93 & 2.90 & 2.77 \\
\hline & GZ8126 & 2.14 & 2.22 & 2.37 & 2.42 & 2.51 & 3.29 & 2.83 & 2.70 & 2.62 \\
\hline & GZ7769 & 2.65 & 2.64 & 2.71 & 2.97 & 2.96 & 3.08 & 2.81 & 2.66 & 2.56 \\
\hline & GZ7764 & 2.30 & 2.36 & 2.52 & 2.53 & 2.72 & 2.66 & 2.54 & 2.50 & 2.50 \\
\hline & GZ6522 & 2.73 & 2.78 & 2.84 & 3.09 & 3.43 & 3.36 & 3.18 & 3.06 & 2.99 \\
\hline & LSD at 0.05 & \multicolumn{9}{|c|}{0.08} \\
\hline \multirow{9}{*}{2010} & GZ8455 & 2.64 & 2.83 & 3.68 & 3.41 & 3.59 & 4.01 & 3.87 & 3.79 & 3.75 \\
\hline & GZ8450 & 2.80 & 3.00 & 3.30 & 3.60 & 3.70 & 3.78 & 3.66 & 3.64 & 3.50 \\
\hline & GZ7576 & 2.77 & 3.14 & 3.47 & 3.58 & 3.99 & 3.52 & 3.42 & 3.33 & 3.30 \\
\hline & GZ8479 & 2.95 & 2.93 & 3.13 & 3.45 & 3.56 & 3.45 & 3.36 & 3.35 & 3.34 \\
\hline & GZ8126 & 3.17 & 3.25 & 3.51 & 3.54 & 3.75 & 3.61 & 3.50 & 3.40 & 2.97 \\
\hline & GZ7769 & 3.00 & 3.02 & 3.18 & 3.34 & 3.49 & 3.80 & 3.71 & 3.65 & 3.44 \\
\hline & GZ7764 & 2.84 & 2.99 & 3.44 & 3.65 & 3.67 & 3.64 & 3.57 & 3.48 & 3.34 \\
\hline & GZ6522 & 3.50 & 3.75 & 3.80 & 3.97 & 4.16 & 3.71 & 3.67 & 3.22 & 3.23 \\
\hline & LSD at 0.05 & \multicolumn{9}{|c|}{0.28} \\
\hline
\end{tabular}

\section{Number of grains panicle ${ }^{-1}$}

Genotypes differed significantly in their number of grains panicle ${ }^{-1}$ (Table 3). Averaged across N levels, GZ8455 had the highest number of grains panicle ${ }^{-1}$. The differences between GZ8455 and GZ6522 were not significant in number of grains panicle ${ }^{-1}$. Generally the application of nitrogen fertilizer caused an increase in number of grains panicles ${ }^{-1}$ compared with the control (Table 3). Data indicated that nitrogen levels significantly affected number of grains panicle-1. Increasing nitrogen levels from 0 to 160 and 200 $\mathrm{kg} \mathrm{N} \mathrm{ha}^{-1}$ increased significantly number of grains panicle- ${ }^{-1}$ in the first and second seasons, respectively. Moreover, there was no significant difference between applying 160 and $200 \mathrm{~kg} \mathrm{~N} \mathrm{ha}^{-1}$. This results mainly due to the fact that the application of nitrogen at panicle initiation was found to be more efficient and used to increase spikelets number. These results supported with that obtained by Ebaid and Ghanem (2000) and Singh et al. (2004).

Concerning the effect of interaction, data in Table 7 showed that number of grains panicle-1 was significantly affected by the interaction between genotypes and nitrogen fertilizer levels. GZ6522 produced the 
maximum number of grains per panicle when fertilized by $200 \mathrm{~kg} \mathrm{~N}^{-1}$ while, GZ8479 produced the minimum number of grains per panicle zero nitrogen fertilizer treatment.

Table 7: Number of grains panicle ${ }^{-1}$ as affected by the interaction between rice entries and nitrogen levels in 2009 and 2010 seasons.

\begin{tabular}{|c|c|c|c|c|c|c|c|c|c|c|}
\hline \multirow{2}{*}{ Season } & \multirow{2}{*}{ Entry } & \multicolumn{9}{|c|}{$\mathrm{N} \mathrm{kg} \mathrm{ha}^{-1}$} \\
\hline & & 0 & 40 & 80 & 120 & 160 & 200 & 240 & 280 & 320 \\
\hline \multirow{9}{*}{2009} & GZ8455 & 104.13 & 121.80 & \begin{tabular}{|l|}
120.40 \\
\end{tabular} & 145.93 & 164.87 & 163.20 & 158.79 & 149.91 & 156.60 \\
\hline & GZ8450 & 92.47 & 118.20 & 135.20 & 141.07 & 155.60 & 138.87 & 128.93 & 113.20 & 96.13 \\
\hline & GZ7576 & 82.80 & 97.27 & 110.40 & 121.13 & 117.07 & 115.24 & 120.47 & 102.20 & 106.87 \\
\hline & GZ8479 & 72.80 & 83.73 & 93.00 & 112.80 & 126.07 & 147.53 & 168.67 & 150.11 & 124.27 \\
\hline & GZ8126 & 104.40 & 118.33 & 134.33 & 143.13 & 153.33 & 165.67 & 142.13 & 150.80 & 154.47 \\
\hline & GZ7769 & 96.40 & 111.40 & 133.80 & 144.27 & 165.13 & 152.33 & 150.27 & 151.80 & 146.33 \\
\hline & GZ7764 & 96.87 & 120.47 & 108.93 & 116.00 & 143.47 & 117.20 & 109.80 & 111.47 & 110.47 \\
\hline & GZ6522 & 106.00 & 128.30 & 138.07 & 150.36 & 160.87 & 173.73 & 146.20 & 137.47 & 134.47 \\
\hline & LSD at 0.05 & \multicolumn{9}{|c|}{4.47} \\
\hline \multirow{9}{*}{2010} & GZ8455 & 121.41 & 133.96 & 137.15 & \begin{tabular}{|l|}
165.46 \\
\end{tabular} & 167.80 & 169.72 & 170.95 & 160.40 & 151.86 \\
\hline & GZ8450 & 115.04 & 132.87 & 148.67 & 158.04 & 166.27 & 155.67 & 146.76 & 142.60 & 140.17 \\
\hline & GZ7576 & 113.20 & 117.50 & 125.10 & 125.41 & 131.70 & 131.10 & 134.00 & 120.05 & 118.80 \\
\hline & GZ8479 & 95.30 & 102.06 & 119.00 & 136.16 & 148.50 & 151.90 & 113.66 & 113.16 & 109.50 \\
\hline & GZ8126 & 122.73 & 130.90 & 141.60 & 152.50 & 155.19 & 156.19 & 145.95 & 145.86 & 142.03 \\
\hline & GZ7769 & 130.86 & 140.32 & 147.00 & 153.46 & 156.24 & 157.98 & 144.01 & 140.66 & 137.56 \\
\hline & GZ7764 & 111.90 & 106.40 & 120.40 & 124.35 & 124.46 & 136.50 & 148.90 & 126.40 & 126.80 \\
\hline & GZ6522 & 128.40 & 130.60 & 135.84 & 143.43 & 170.60 & 173.51 & 171.08 & 154.60 & 150.20 \\
\hline & LSD at 0.05 & \multicolumn{9}{|c|}{10.70} \\
\hline
\end{tabular}

\section{Sterility \%}

The tested rice genotypes and nitrogen levels had significant effect in sterility percentage in both seasons (Table 3). The highest sterility percentage was found in GZ8479 followed by GZ8455. This could be due to genetic background. Sterility percentage gradually increased as nitrogen level increased up to320 $\mathrm{kg} \mathrm{N} \mathrm{ha}^{-1}$. These results were true in both seasons. The increase in spikelet sterility with increasing $\mathrm{N}$ levels may be associated with more spikelets produced per plant with increasing $N$ levels and photoassimilate produced by source may not be sufficient to fill large number of spikelets. In other words, there was no appropriate balance between source and sink.

Data in Table 8 revealed that the highest sterility \% was obtained when GZ8479 was fertilized by $320 \mathrm{~kg} \mathrm{~N}^{-1}$ both seasons. On the other hand the lowest sterility \% were produced from GZ7769 in 2009 season and GZ8126 in 2010 season under unfertilized plots. 
Metwally, T. F. et al.

Table 8: Sterility \% as affected by the interaction between rice entries and nitrogen levels in 2009 and 2010 seasons.

\begin{tabular}{|c|c|c|c|c|c|c|c|c|c|c|}
\hline \multirow{2}{*}{ Season } & \multirow{2}{*}{ Entry } & \multicolumn{9}{|c|}{ N kg ha ${ }^{-1}$} \\
\hline & & 0 & 40 & 80 & 120 & 160 & 200 & 240 & 280 & 320 \\
\hline \multirow{9}{*}{2009} & GZ8455 & 4.71 & 5.50 & 5.85 & 5.78 & 5.74 & 6.44 & 6.68 & 8.04 & 12.37 \\
\hline & GZ8450 & 2.67 & 3.02 & 3.38 & 3.55 & 4.35 & 4.97 & 6.77 & 8.54 & 11.41 \\
\hline & GZ7576 & 1.97 & 3.19 & 2.87 & 2.87 & 3.13 & 3.57 & 3.91 & 5.55 & 7.36 \\
\hline & GZ8479 & 4.50 & 4.66 & 4.71 & 4.79 & 4.89 & 5.71 & 8.00 & 11.87 & 12.81 \\
\hline & GZ8126 & 2.75 & 3.78 & 3.73 & 3.07 & 2.78 & 2.37 & 3.38 & 4.12 & 7.81 \\
\hline & GZ7769 & 1.91 & 2.39 & 2.67 & 3.12 & 2.74 & 3.92 & 4.29 & 6.39 & 6.74 \\
\hline & GZ7764 & 2.75 & 2.94 & 3.33 & 3.68 & 4.32 & 5.01 & 5.68 & 6.25 & 6.97 \\
\hline & GZ6522 & 2.74 & 3.11 & 3.36 & 2.86 & 3.67 & 3.19 & 4.54 & 4.91 & 5.60 \\
\hline & LSD at 0.05 & \multicolumn{9}{|c|}{0.45} \\
\hline \multirow{9}{*}{2010} & GZ8455 & 2.99 & 4.40 & 5.47 & 4.73 & 5.08 & 5.97 & 6.26 & 8.86 & 10.80 \\
\hline & GZ8450 & 3.49 & 4.22 & 3.70 & 3.77 & 3.49 & 5.24 & 7.02 & 7.54 & 9.26 \\
\hline & GZ7576 & 2.16 & 2.51 & 2.94 & 3.94 & 3.70 & 4.68 & 6.40 & 8.70 & 8.97 \\
\hline & GZ8479 & 6.38 & 7.03 & 6.14 & 6.87 & 6.98 & 7.81 & 9.42 & 9.43 & 13.50 \\
\hline & GZ8126 & 1.43 & 1.80 & 1.98 & 1.85 & 2.37 & 2.79 & 3.64 & 4.25 & 6.26 \\
\hline & GZ7769 & 2.20 & 2.58 & 3.92 & 4.04 & 4.17 & 4.70 & 5.09 & 7.73 & 10.43 \\
\hline & GZ7764 & 2.64 & 3.28 & 3.03 & 3.62 & 4.89 & 5.37 & 5.04 & 6.27 & 6.80 \\
\hline & GZ6522 & 2.53 & 2.15 & 2.03 & 3.05 & 2.98 & 3.21 & 3.82 & 3.84 & 4.57 \\
\hline & LSD at 0.05 & \multicolumn{9}{|c|}{0.68} \\
\hline
\end{tabular}

\section{0-grain weight $(g)$ :}

Data regarding 1000-grain weight are shown in Table 9 and 10. Significant variation among the tested genotypes were observed. Among the genotypes, GZ6522 produced the highest values of 1000-grain weight followed by GZ8126 and GZ8479. But GZ8450 gave the lowest values in this respect. The weight of 1000 -grain significantly influenced by nitrogen application. Zero, 160 and $200 \mathrm{~kg} \mathrm{~N}^{-1}$ produced the maximum values of 1000 -grain weight without any significant differences among them. Increasing nitrogen levels from 200 up to $320 \mathrm{~N} \mathrm{ha}^{-1}$ decreased significantly the weight of 1000 -grain. This mainly due to that nitrogen increased spikelets number at high nitrogen levels. In contrast plants can not produce enough carbohydrates to fill all spikelets produced under high nitrogen fertilization level. These results are similar to those obtained by Mauad, et al. (2003) and Ghanbari-Malidareh (2011).

Interaction effects between $\mathrm{N}$ levels and entries on 1000-grain weight were significant in both seasons (Table 10). The maximum values of 1000 grain weight were observed where GZ6522 was received 160, 200 or $240 \mathrm{~kg}$ $\mathrm{N}$ ha-1. 
Table 9: 1000-grain weight, grain yield, straw yield and harvest index of rice entries as affected by nitrogen levels in 2009 and 2010 seasons.

\begin{tabular}{|c|c|c|c|c|c|c|c|c|}
\hline \multirow{2}{*}{ Treatment } & \multicolumn{2}{|c|}{1000 grain weight $\mathrm{g}$} & \multicolumn{2}{|c|}{ Grain yield $\mathrm{t} \mathrm{ha} \mathbf{~}^{-1}$} & \multicolumn{2}{|c|}{ Straw yield $\mathrm{t} \mathrm{ha}^{-1}$} & \multicolumn{2}{|c|}{ Harvest Index } \\
\hline & 2009 & 2010 & 2009 & 2010 & 2009 & 2010 & 2009 & 2010 \\
\hline Entry: & & & & & & & & \\
\hline GZ8455 & 24.23 & 24.51 & 8.40 & 9.64 & 10.23 & 11.09 & 45.04 & 46.54 \\
\hline GZ8450 & 22.98 & 23.43 & 7.73 & 9.03 & 10.40 & 11.43 & 42.90 & 44.18 \\
\hline GZ7576 & 24.42 & 24.57 & 7.69 & 8.69 & 10.15 & 11.94 & 43.19 & 42.12 \\
\hline GZ8479 & 25.32 & 25.98 & 7.77 & 8.42 & 10.31 & 11.54 & 43.15 & 42.20 \\
\hline GZ8126 & 25.74 & 26.32 & 7.80 & 9.54 & 10.11 & 12.59 & 43.53 & 43.02 \\
\hline GZ7769 & 23.85 & 24.71 & 7.52 & 9.86 & 9.63 & 11.22 & 43.94 & 46.90 \\
\hline GZ7764 & 23.95 & 24.48 & 7.05 & 8.46 & 9.92 & 11.29 & 41.71 & 42.79 \\
\hline GZ6522 & 26.63 & 27.13 & 8.83 & 10.38 & 11.44 & 12.69 & 43.72 & 45.03 \\
\hline LSD at 0.05 & 0.33 & 0.37 & 0.12 & 0.19 & 0.09 & 0.17 & 0.45 & 0.52 \\
\hline N kg ha-1 : & & & & & & & & \\
\hline 0 & 26.00 & 26.28 & 5.97 & 7.59 & 7.47 & 9.77 & 44.36 & 43.69 \\
\hline 40 & 25.02 & 25.78 & 6.89 & 8.36 & 8.60 & 10.34 & 44.48 & 44.67 \\
\hline 80 & 24.11 & 24.90 & 7.44 & 8.84 & 9.37 & 10.90 & 44.23 & 44.69 \\
\hline 120 & 24.50 & 24.40 & 8.14 & 9.34 & 9.94 & 11.37 & 45.01 & 45.05 \\
\hline 160 & 25.70 & 26.59 & 8.74 & 10.12 & 10.60 & 12.01 & 45.13 & 45.70 \\
\hline 200 & 25.90 & 26.22 & 8.82 & 10.14 & 11.08 & 12.19 & 44.30 & 45.39 \\
\hline 240 & 24.28 & 25.17 & 8.50 & 9.96 & 11.41 & 12.67 & 42.69 & 43.97 \\
\hline 280 & 23.58 & 24.12 & 8.27 & 9.68 & 11.81 & 13.12 & 41.20 & 42.44 \\
\hline 320 & 22.71 & 22.82 & 7.87 & 9.24 & 12.21 & 13.14 & 39.18 & 41.28 \\
\hline LSD at 0.05 & 0.30 & 0.31 & 0.15 & 0.19 & 0.12 & 0.14 & 0.50 & 0.52 \\
\hline Interaction & $\star \star$ & $\star \star$ & $\star \star *$ & $\star \star$ & $\star \star$ & $\star \star$ & * & $\star \star$ \\
\hline
\end{tabular}

Table 10: 1000-grain weight as affected by the interaction between rice entries and nitrogen levels in 2009 and 2010 seasons.

\begin{tabular}{|c|c|c|c|c|c|c|c|c|c|c|}
\hline \multirow{2}{*}{ Season } & \multirow{2}{*}{ Entry } & \multicolumn{9}{|c|}{ N kg ha ${ }^{-1}$} \\
\hline & & 0 & 40 & 80 & 120 & 160 & 200 & 240 & 280 & 320 \\
\hline \multirow{9}{*}{2009} & GZ8455 & 25.73 & 25.87 & 25.88 & 24.19 & 26.44 & 24.03 & 22.44 & 21.95 & 21.54 \\
\hline & GZ8450 & 23.42 & 22.77 & 21.87 & 23.78 & 25.16 & 24.13 & 22.87 & 22.12 & 20.73 \\
\hline & GZ7576 & 25.79 & 25.25 & 23.32 & 23.24 & 24.06 & 26.21 & 25.11 & 23.78 & 23.01 \\
\hline & GZ8479 & 26.99 & 25.42 & 25.49 & 24.37 & 25.96 & 25.73 & 25.62 & 24.30 & 23.97 \\
\hline & GZ8126 & 27.42 & 25.32 & 24.70 & 26.69 & 26.82 & 27.61 & 24.33 & 24.85 & 23.88 \\
\hline & GZ7769 & 25.79 & 25.23 & 22.67 & 23.69 & 23.95 & 25.32 & 23.29 & 23.17 & 21.51 \\
\hline & GZ7764 & 24.83 & 23.88 & 23.22 & 24.88 & 25.41 & 26.07 & 22.74 & 22.53 & 22.00 \\
\hline & GZ6522 & 27.66 & 26.41 & 25.76 & 25.17 & 27.77 & 28.08 & 27.80 & 25.96 & 25.08 \\
\hline & LSD at 0.05 & \multicolumn{9}{|c|}{0.85} \\
\hline \multirow{9}{*}{2010} & GZ8455 & 25.31 & 26.00 & 24.80 & 24.02 & 26.61 & 24.06 & 23.76 & 22.77 & 22.73 \\
\hline & GZ8450 & 24.14 & 23.87 & 23.08 & 23.17 & 24.91 & 24.65 & 23.62 & 22.72 & 20.75 \\
\hline & GZ7576 & 26.14 & 25.70 & 23.88 & 22.75 & 26.09 & 26.25 & 24.17 & 23.93 & 22.28 \\
\hline & GZ8479 & 26.58 & 26.00 & 25.84 & 24.67 & 27.01 & 27.01 & 26.67 & 25.51 & 24.53 \\
\hline & GZ8126 & 27.59 & 27.02 & 26.07 & 26.45 & 27.35 & 27.16 & 26.27 & 25.35 & 23.62 \\
\hline & GZ7769 & 26.27 & 25.61 & 25.14 & 24.11 & 26.32 & 26.01 & 24.86 & 22.94 & 21.14 \\
\hline & GZ7764 & 26.39 & 25.33 & 24.30 & 23.82 & 26.13 & 25.00 & 23.60 & 23.16 & 22.60 \\
\hline & GZ6522 & 27.81 & 26.74 & 26.08 & 26.21 & 28.30 & 29.08 & 28.46 & 26.59 & 24.92 \\
\hline & LSD at 0.05 & \multicolumn{9}{|c|}{0.89} \\
\hline
\end{tabular}

Grain yield ( $t$ ha $\left.^{-1}\right)$ :

Grain yield of eight rice genotypes as influenced by the application of different nitrogen levels and their interaction are presented in Tables 9 and 11. There was significant variation among various genotypes in grain yield. Maximum grain yield was produced by GZ6522 rice line followed by GZ8455. 
While GZ7764 genotype produced the lowest grain yield as compared with the other rice genotypes. Data in Table 9 showed that the grain yield was significantly affected by nitrogen application. Nitrogen application increased the yield of all eight genotypes as compare with no $\mathrm{N}$ application treatment. It might be due to the effect of nitrogen on the proliferation of roots so the uptake level from soil was increased and supplying the upground parts of the plant. Data showed also that significant increase in grain yield as nitrogen levels increased from 0 up to $200 \mathrm{~kg} \mathrm{~N} \mathrm{ha}^{-1}$ in the first and second seasons. The difference in grain yield between 160 and $200 \mathrm{~kg} \mathrm{~N}^{-1}$ was not significant. The increase in grain yield by increasing nitrogen level from 0 up to $200 \mathrm{~kg} \mathrm{~N} \mathrm{ha}^{-1}$ was due to the increase in most of yield components as a result to establish optimal canopy which led to produced sufficient photosynthetic products consequently increase filling and gave greatest grain yield. Similar results were previously drawn by Singh et al. (2004), Mhaskar et al. (2005) and Fageria et al. (2006) who stated that optimal yield may be achieved by successful regulation of source-sink relationships for production and utilization of photoassimilate within plants.

The interaction effect between genotypes and $\mathrm{N}$ fertilizer levels on grain yield was significant. The maximum grain yield was observed in GZ6522 when fertilized with 160 or $200 \mathrm{~kg} \mathrm{~N}^{-1}$ in both seasons while, the minimum grain yield were recorded by GZ7764 in the first season and GZ8479 and GZ7764 in the second season under control plots.

Table 11: Grain yield as affected by the interaction between rice entries and nitrogen levels in 2009 and 2010 seasons.

\begin{tabular}{|c|c|c|c|c|c|c|c|c|c|c|}
\hline \multirow{2}{*}{ Season } & \multirow{2}{*}{ Entry } & \multicolumn{9}{|c|}{$\mathrm{N} \mathrm{kg} \mathrm{ha}^{-1}$} \\
\hline & & 0 & 40 & 80 & 120 & 160 & 200 & 240 & 280 & 320 \\
\hline \multirow{9}{*}{2009} & GZ8455 & 5.97 & 6.78 & 7.52 & 8.62 & 9.96 & 9.64 & 8.89 & 9.23 & 9.01 \\
\hline & GZ8450 & 6.84 & 7.17 & 7.45 & 8.32 & 8.09 & 8.02 & 8.00 & 7.99 & 7.72 \\
\hline & GZ7576 & 6.40 & 6.70 & 6.70 & 7.79 & 8.16 & 8.24 & 8.56 & 8.46 & 8.24 \\
\hline & GZ8479 & 6.25 & 7.13 & 7.47 & 7.78 & 8.90 & 8.89 & 8.30 & 7.88 & 7.29 \\
\hline & GZ8126 & 5.66 & 6.75 & 7.56 & 7.91 & 8.48 & 9.11 & 9.03 & 7.91 & 7.77 \\
\hline & GZ7769 & 5.50 & 6.45 & 7.22 & 7.70 & 8.39 & 8.61 & 8.11 & 7.96 & 7.78 \\
\hline & GZ7764 & 5.00 & 6.57 & 6.83 & 7.40 & 7.51 & 7.92 & 7.76 & 7.75 & 6.75 \\
\hline & GZ6522 & 6.10 & 7.60 & 8.79 & 9.62 & 10.46 & 10.13 & 9.31 & 9.00 & 8.43 \\
\hline & LSD at 0.05 & \multicolumn{9}{|c|}{0.41} \\
\hline \multirow{9}{*}{2010} & GZ8455 & 8.02 & 8.97 & 9.19 & 9.63 & 10.48 & 10.76 & 10.10 & 10.02 & 9.55 \\
\hline & GZ8450 & 7.90 & 8.53 & 8.64 & 9.47 & 9.63 & 9.87 & 9.27 & 9.04 & 8.88 \\
\hline & GZ7576 & 7.38 & 7.58 & 7.83 & 8.89 & 9.23 & 9.45 & 9.55 & 9.25 & 9.03 \\
\hline & GZ8479 & 6.59 & 7.55 & 7.80 & 7.94 & 9.67 & 9.24 & 9.39 & 8.97 & 8.65 \\
\hline & GZ8126 & 7.45 & 8.02 & 9.59 & 9.98 & 10.33 & 10.39 & 10.52 & 10.11 & 9.48 \\
\hline & GZ7769 & 8.86 & 9.43 & 9.72 & 10.15 & 10.79 & 10.44 & 10.13 & 9.95 & 9.30 \\
\hline & GZ7764 & 6.60 & 7.66 & 7.86 & 8.05 & 9.10 & 9.45 & 9.49 & 9.29 & 8.62 \\
\hline & GZ6522 & 7.95 & 9.10 & 10.06 & 10.59 & 11.73 & 11.53 & 11.21 & 10.83 & 10.43 \\
\hline & LSD at 0.05 & \multicolumn{9}{|c|}{0.53} \\
\hline
\end{tabular}

\section{Straw yield}

Straw yield also significantly differ among the genotypes (Table 9). The highest straw yield was produced in GZ6522. Data in Table 9 also showed that straw yield gradually increased as nitrogen level increased up to $320 \mathrm{~kg} \mathrm{~N} \mathrm{ha}^{-1}$. Minimum straw yield was observed in control. 
The performance of genotypes significantly varied according to nitrogen application. GZ6522 recorded the highest straw yield when fertilized with $320 \mathrm{~kg} \mathrm{~N} \mathrm{ha}^{-1}$ in both seasons (Table 12). Chaturvedi (2005) reported that nitrogen nutrition influences the content of photosynthetic pigments, the biosynthesis of the enzymes taking part in the carbon reduction. Thus the increase in growth and yield owing to the application of $\mathrm{N}$-fertilizers may be attributed to the fact that these nutrients being important constituents of proteins, chlorophyll and enzymes, involve in various metabolic processes which have direct impact on vegetative and reproductive phases of plants.

Table 12: Straw yield as affected by the interaction between rice entries and nitrogen levels in 2009 and 2010 seasons.

\begin{tabular}{|c|c|c|c|c|c|c|c|c|c|c|}
\hline \multirow{2}{*}{ Season } & \multirow{2}{*}{ Entry } & \multicolumn{9}{|c|}{ N kg ha-1 } \\
\hline & & 0 & 40 & 80 & 120 & 160 & 200 & 240 & 280 & 320 \\
\hline \multirow{9}{*}{2009} & GZ8455 & 7.73 & 8.68 & 8.78 & 9.81 & 10.81 & 11.21 & 11.41 & 11.84 & 11.82 \\
\hline & GZ8450 & 7.81 & 8.57 & 9.31 & 10.34 & 10.37 & 10.78 & 11.70 & 12.11 & 12.61 \\
\hline & GZ7576 & 7.77 & 8.83 & 9.07 & 10.01 & 10.77 & 10.86 & 11.01 & 11.37 & 11.63 \\
\hline & GZ8479 & 7.38 & 8.44 & 9.43 & 10.37 & 10.11 & 11.37 & 11.66 & 11.86 & 12.19 \\
\hline & GZ8126 & 7.88 & 9.09 & 9.24 & 9.42 & 10.02 & 10.72 & 10.77 & 11.53 & 12.33 \\
\hline & GZ7769 & 6.87 & 8.00 & 9.72 & 8.90 & 9.91 & 10.25 & 10.62 & 10.92 & 11.46 \\
\hline & GZ7764 & 6.67 & 8.39 & 9.00 & 9.62 & 10.42 & 10.68 & 11.01 & 11.38 & 12.15 \\
\hline & GZ6522 & 7.61 & 8.80 & 10.41 & 11.02 & 12.39 & 12.73 & 13.11 & 13.44 & 13.46 \\
\hline & LSD at 0.05 & \multicolumn{9}{|c|}{0.34} \\
\hline \multirow{9}{*}{2010} & GZ8455 & 9.49 & 9.50 & 10.31 & 10.24 & 11.99 & 11.85 & 12.09 & 12.20 & 12.14 \\
\hline & GZ8450 & 9.14 & 10.67 & 10.71 & 11.64 & 11.46 & 11.56 & 12.65 & 12.69 & 12.37 \\
\hline & GZ7576 & 10.04 & 10.41 & 11.19 & 11.61 & 12.36 & 12.30 & 12.67 & 13.25 & 13.67 \\
\hline & GZ8479 & 9.75 & 10.18 & 10.20 & 10.50 & 11.43 & 12.27 & 12.25 & 13.68 & 13.59 \\
\hline & GZ8126 & 11.40 & 11.59 & 11.55 & 12.52 & 12.99 & 12.81 & 13.59 & 13.61 & 13.22 \\
\hline & GZ7769 & 9.37 & 9.66 & 10.6. & 10.95 & 11.12 & 11.48 & 12.01 & 12.69 & 13.04 \\
\hline & GZ7764 & 9.60 & 9.93 & 10.46 & 10.62 & 11.35 & 11.59 & 12.58 & 12.74 & 12.80 \\
\hline & GZ6522 & 9.40 & 10.79 & 12.16 & 12.93 & 13.38 & 13.65 & 13.56 & 14.11 & 14.28 \\
\hline & LSD at 0.05 & \multicolumn{9}{|c|}{0.41} \\
\hline
\end{tabular}

\section{Harvest Index}

Genotypes differences, nitrogen levels and their interaction are presented in (Table 9). GZ8455 produced the highest values of harvest index and was superior to other genotypes. GZ7764 and GZ7576 recorded the lowest values in the first and second seasons, respectively. Data showed that significant increase in harvest index as nitrogen levels increased from 0 up to $160 \mathrm{~kg} \mathrm{~N} \mathrm{ha}^{-1}$. A significant reduction in harvest index was happened when nitrogen levels increased from 240 to $320 \mathrm{~kg} \mathrm{~N} \mathrm{ha}^{-1}$.

With regard to the interaction effect, application of 120 or $160 \mathrm{~kg} \mathrm{~N}$ ha${ }^{1}$ with GZ8455 in the first season and with GZ7769 in the second season caused the highest values of harvest index. GZ7764 and GZ8479 at $320 \mathrm{~kg} \mathrm{~N}$ $\mathrm{ha}^{-1}$ recorded the lowest values of harvest index in the first and second seasons, respectively (Table 13 ).

Sinclair (1998) stated that harvest index is a critical character associated with dramatic increase in grain yield that have occurred in recent century. This trait actually reflects the partitioning of photosynthate between the grain and vegetative parts of plant and improvements in harvest index emphasize the importance of carbon allocation in grain production. 
Table 13: Harvest Index as affected by the interaction between rice entries and nitrogen levels in 2009 and 2010 seasons.

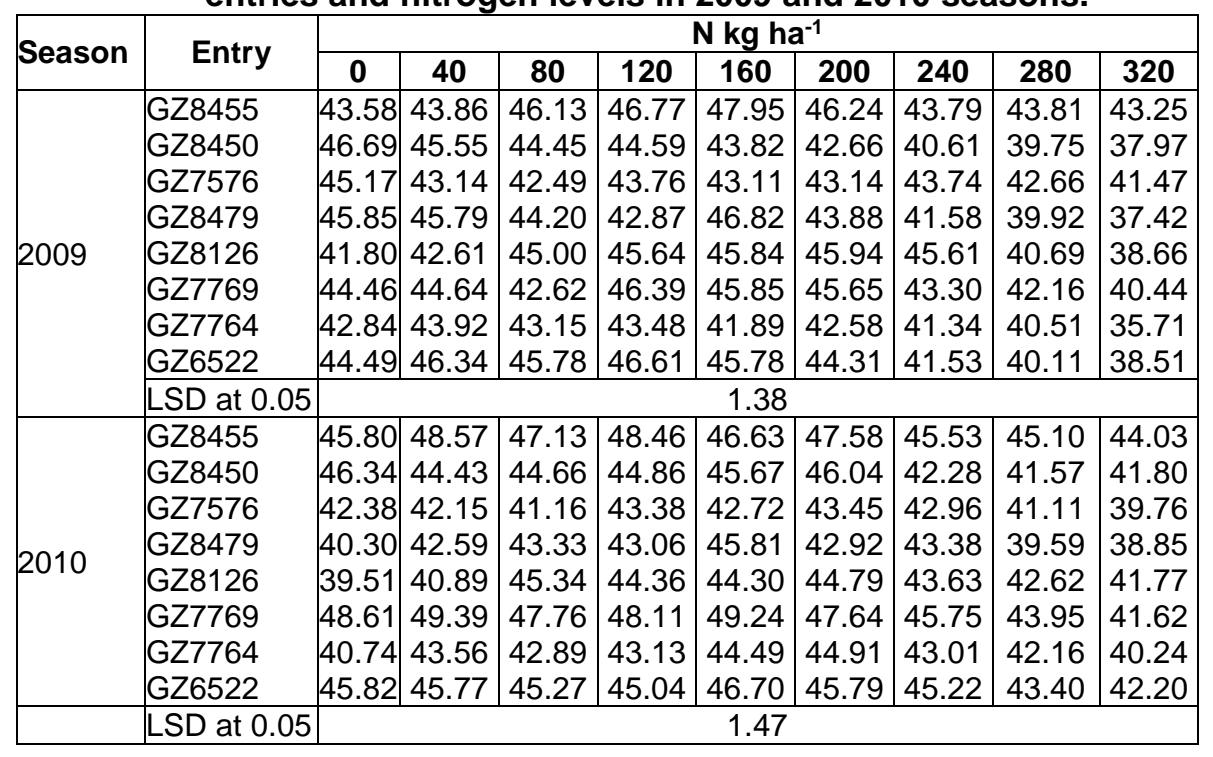

Agronomic Nitrogen Use Efficiency ANUE

Agronomic use efficiency of nitrogen fertilizer is presented in Table 14. Data indicted that ANUE was decrease by increasing $\mathrm{N}$ levels. Eagle et al. (2000) reported that in rice $\mathrm{N}$ use efficiency, which has both a physiological and soil $\mathrm{N}$ supply components, decreased with increase in soil $\mathrm{N}$ supply.

Table 14: Agronomic nitrogen use efficiency (ANUE) of rice entries as affected by nitrogen levels in 2009 and 2010 seasons.

\begin{tabular}{|l|l|c|c|c|c|c|c|c|c|}
\hline \multirow{2}{*}{ Season } & \multirow{2}{*}{ Entry } & \multicolumn{7}{|c|}{$\mathbf{N ~ k g ~ h a}^{-1}$} \\
\cline { 3 - 9 } & & $\mathbf{4 0}$ & $\mathbf{8 0}$ & $\mathbf{1 2 0}$ & $\mathbf{1 6 0}$ & $\mathbf{2 0 0}$ & $\mathbf{2 4 0}$ & $\mathbf{2 8 0}$ & $\mathbf{3 2 0}$ \\
\hline \multirow{5}{*}{$\mathbf{2 0 0 9}$} & GZ8455 & 20.25 & 19.38 & 22.08 & 24.94 & 18.35 & 12.17 & 11.64 & 9.50 \\
& GZ8450 & 8.25 & 7.63 & 12.33 & 7.81 & 5.90 & 4.83 & 4.11 & 2.75 \\
& GZ7576 & 7.50 & 3.75 & 11.58 & 11.00 & 9.20 & 9.00 & 7.36 & 5.75 \\
& GZ8479 & 22.00 & 15.25 & 12.75 & 16.56 & 13.20 & 8.54 & 5.82 & 3.25 \\
& GZ8126 & 27.25 & 23.75 & 18.75 & 17.63 & 17.25 & 14.04 & 8.04 & 6.59 \\
& GZ7769 & 23.75 & 21.50 & 18.33 & 18.06 & 15.55 & 10.88 & 8.79 & 7.13 \\
& GZ7764 & 39.25 & 22.88 & 20.00 & 15.69 & 14.60 & 11.50 & 9.82 & 5.47 \\
& GZ6522 & 37.50 & 33.63 & 29.33 & 27.25 & 20.15 & 13.38 & 10.36 & 7.28 \\
\hline \multirow{5}{*}{$\mathbf{2 0 1 0}$} & GZ8455 & 23.75 & 14.63 & 10.06 & 15.38 & 13.70 & 8.67 & 7.14 & 4.78 \\
& GZ8450 & 15.75 & 9.25 & 9.81 & 10.81 & 9.85 & 5.71 & 4.07 & 3.06 \\
& GZ7576 & 5.00 & 5.63 & 9.44 & 11.56 & 10.35 & 9.04 & 6.68 & 5.16 \\
& GZ8479 & 24.00 & 15.13 & 8.44 & 19.25 & 13.25 & 11.67 & 8.50 & 6.44 \\
& GZ8126 & 14.25 & 26.75 & 15.81 & 18.00 & 14.70 & 12.79 & 9.50 & 6.34 \\
& GZ7769 & 14.25 & 10.75 & 8.06 & 12.06 & 7.90 & 5.29 & 3.89 & 1.38 \\
& GZ7764 & 26.50 & 15.75 & 9.06 & 15.63 & 14.25 & 12.04 & 9.61 & 6.31 \\
& GZ6522 & 28.75 & 26.38 & 16.50 & 23.63 & 17.90 & 13.58 & 10.29 & 7.75 \\
\hline
\end{tabular}


Agronomic nitrogen use efficiency (ANUE) was varied among genotypes and it ranged from 2.75 to 39.25 and from 1.38 to $28.75 \mathrm{~kg}$ grain produced per $\mathrm{kg}$ of $\mathrm{N}$ applied in the first and second season, respectively. Across $\mathrm{N}$ levels, GZ6522 recorded the highest values of ANUE at all nitrogen levels in the two seasons except at $40 \mathrm{~kg} \mathrm{~N} \mathrm{ha}^{-1}$ in the first season where GZ7764 recorded the highest value. Many researchers have reported significant variations of $\mathrm{N}$-use efficiency among lowland rice genotypes (Fageria and Baligar 2003). Such differences may be related to genetic factors, physiological processes (absorption, translocation, assimilation, $\mathrm{N}$ remobilization, and storage), and biochemical processes (enzyme nitrate reductase efficiency) (Isfan, 1993 and Fageria and Baligar 2003).

\section{Conclusion}

Among the tested rice genotypes, GZ6522 (Sakha105) had higher response to nitrogen fertilization than the other tested genotypes while, GZ7764 had the less responded. The optimum dosage of nitrogen fertilizer for the tested genotypes was $160 \mathrm{~kg} \mathrm{~N} \mathrm{ha}^{-1}$. GZ6522 (Sakha105) was the best in grain yield which produced 10.46 and $11.73 \mathrm{t} \mathrm{ha}^{-1}$ in 2009 and 2010 seasons, respectively.

\section{REFERENCES}

Chaturvedi, I. (2005). Effect of nitrogen fertilizers on growth, yield and quality of hybrid rice (Oryza sativa L.). J. of Central European Agric. 6(4):611618.

Eagle, A.J.; J.A Bird, W.R Horwath, B.A. Linquist, S.M. Brouder, J.E. Hill and C.V. Kessel (2000). Rice yield and nitrogen utilization efficiency under alternative straw management practices. Agron. J., 92, 1096-1103.

Ebaid, R.A. and S.A. Ghanem (2000). Productivity of Giza177 rice variety grown after different winter crops and fertilized with different nitrogen levels. Egypt. J. Agric. Res. 78(2):717-731.

Fageria, N. K.; V. C. Baligar and R. B. Clark (2006). Physiology of Crop Production. New York: The Haworth Press.(C.F. Computer Search).

Fageria, N.K. and V.C. Baligar (2003). Methodology for evaluation of lowland rice genotypes for nitrogen use efficincy. J. Plant Nutr., 26:1315-1333.

FAO (Food and Agriculture Organization) (2004). Selected indicators of FAO in Asia-pacific region corporate document repository. pp. 87-91.

Ghanbari-Malidareh, Abbas (2011). Silicon application and nitrogen on yield and yield Components in rice (Oryza sativa L.) in two irrigation systems. World Academy of Science, Engineering and Technology, 74:88-95.(C.F. Computer Search).

Gomez, K.A. and A.A. Gomez. 1984. Statistical procedure for agicultural research. $2^{\text {nd }}$ Ed. Johin Wiley Sons. New yourk. USA.

Isfan, D. (1993). Genotypic variability for physiological efficiency index of nitrogen in oats. Plant Soil, 154: 53-59.

Mauad, M.; C.A.C. Crusciol; H. Grassi Filho and J.C. Correa (2003). Nitrogen and silicon fertilization of upland rice. Scientia Agricola, 60:761-765. 
Meisinger, J.J.; J.S. Schepers, and W.R. Raun (2008). Crop nitrogen requirement and fertilization. p. 563-612. In J.S. Schepers and W.R. Raun (ed.) Nitrogen in agricultural systems. Agron. Monogr., 49. ASA and SSSA, Madison, WI.

Metwally, T.F.; S.E.M. Sedeek; A.F. Abdelkhalik;, I.M. El-Rewiny and E.M.R. Metwali (2010). Genetic behaviour of some rice (Oryza sativa L.) genotypes under different treatments of nitrogen levels. AmericanEurasian J. Agric. \& Environ. Sci., 8 (1): 27-34.

Mhaskar,N.V.; J.H.Dongale; A.A. Dademal, and S.A. Khanvilkar (2005). Performance of scented rice varieties under different nitrogen levels in lateritic soil of Konkan Oryza, 42(4): 323-326.

Saleque M.A.; U.A. Naher; N.N. Choudhury, and A.T.M.S. Hossain (2004). Variety-specific nitrogen fertilizer recommendation for lowland rice. Communications in Soil Science and Plant Analysis, 35:1891-1903.

Shaiful Islam, Md.; M. Hasanuzzamanb; M. Rokonuzzaman and K. Nahard (2009). Effect of split application of nitrogen fertilizer on morphophysiological parameters of rice genotypes. International J. of Plant Production, 3(1): 51-62

Sinclair, T.R. (1998). Histrotical changes in harvest index and crop nitrogen accumulation. Crop Sci., 38:638-643.

Singh, T.; Y.S. Shivay and S. Singh (2004). Effect of date of transplanting and nitrogen on productivity and nitrogen use indices in hybrid and non-hybrid aromatic rice. Acta Agronomica Hungarica, 52(3): 245-252. (C.F. Computer Search).

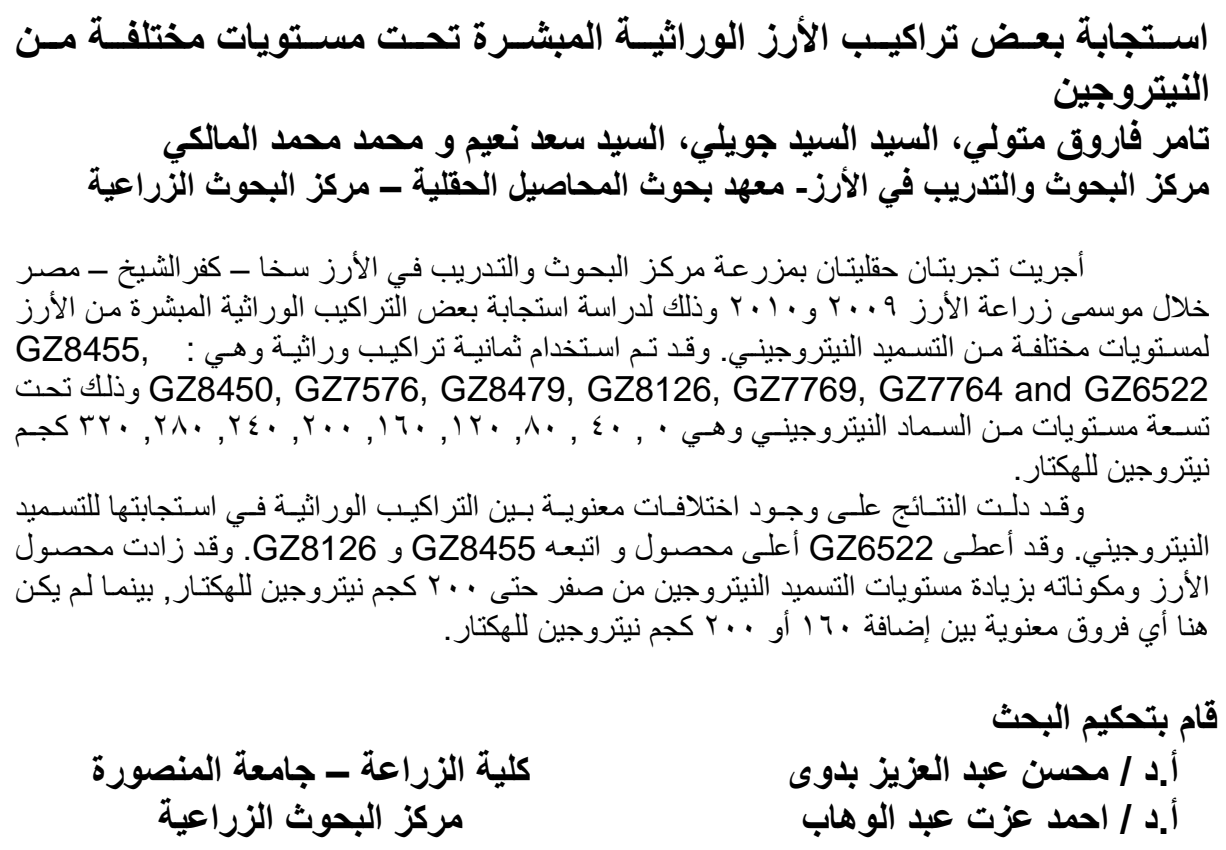

